\title{
论 文 群体智能及工程应用专辑
}

\section{基于增益矩阵的二阶集群系统鲁棒分布式立体 编队控制}

\author{
徐扬, 罗德林 ${ }^{*}$, 周路鹏, 邵将, 尤延铖 \\ 厦门大学航空航天学院, 厦门 361102 \\ *E-mail: luodelin1204@xmu.edu.cn
}

收稿日期: 2019-07-26; 接受日期: 2019-09-05; 网络版发表日期: 2020-02-20

国家自然科学基金(批准号: 61673327)、航空电子系统综合技术重点实验室和航空科学基金(编号: 20185568005)资助项目

\begin{abstract}
摘要 本文研究解决了在三维空间内针对二阶集群系统的鲁棒分布式编队生成与保持控制问题, 所设计的基于 增益矩阵的控制方法仅需测量局部坐标系下智能体间的相对位置信息而不依赖于交互通信。首先设计了二阶集 群系统的立体编队生成控制策略, 通过构造李雅普诺夫函数证明了全局稳定性, 并利用凸优化求解得出增益矩 阵. 所设计的生成控制律对扰动、输入饱和等具有较好的鲁棒性，同时在生成过程中引入了启发式的避碰算法. 在此基础上，设计了带有期望距离的编队保持控制策略，使得集群系统能够保持固定大小的队形。然后，将所设 计的控制律进行改进，拓展并应用于领航者-跟随者网络下的二阶集群系统立体编队控制。最后，通过相关的仿真 实验验证了理论结果的正确性.
\end{abstract}

关键词 分布式编队控制, 二阶集群系统, 鲁棒性, 避碰, 领航者-跟随者网络

\section{1 引言}

近年来，技术的进步使得人们能够通过部署大规 模的智能体如无人机、无人车等组成集群系统，来执 行环境测绘 ${ }^{[1]}$ 及监察 ${ }^{[2]}$ 、货物运输 ${ }^{[3]}$ 、对象操纵 ${ }^{[4 \sim 6]}$ 等 任务. 在这些实际应用中, 集群系统能够自主实现所需 的编队队形是构建更复杂的机动和导航策略的基础. 关于集群系统的编队控制, 目前已有大量的研究工 作 ${ }^{[7]}$, 其主要的研究内容可以分为队形生成、队形保 持、队形切换、编队避障、自适应等.

集群系统的编队控制策略主要分为两种：集中式
编队控制策略和分布式编队控制策略. 随着执行任务 的复杂化，所需部署的智能体个数也需不断增加. 由 于受到通信带宽的限制，智能体间的通信带宽会逐渐 趋于饱和，这使得传统的集中式控制方法变得越来越 不实用. 另外在一些场合，如室内、水下、深空中， GPS位置测量可能会失效，导致智能体获取不到其他 智能体在全局坐标系里的状态信息，这时也不能采用 集中式的编队控制方法. 此外，集中式控制具有缺乏 对通信丢失、硬件故障、对通信信号的干扰或欺骗等 恶意攻击的恢复能力以及对全局测量的鲁棒性等缺 点. 基于这些缺陷, 分布式的编队控制策略得到了广泛

引用格式: 徐扬, 罗德林, 周路鹏, 等. 基于增益矩阵的二阶集群系统鲁棒分布式立体编队控制. 中国科学: 技术科学, 2020, 50: 461-474

Xu Y, Luo D L, Zhou L P, et al. A gain matrix approach for robust distributed 3D formation control with second order swarm systems (in Chinese). Sci Sin Tech, 2020, 50: 461-474, doi: 10.1360/SST-2019-0258 
的关注与研究. 通过将局部控制律分配给单个智能体, 确保了从智能体的集体行为中产生期望的几何形状. 分布式的编队控制可以应用到很多场合，与集中式编 队控制相比，分布式控制策略大大降低了对智能体通 讯能力的要求, 具有更好的可扩展性、自然的并行计 算能力、对通信损耗和硬件故障的恢复能力、对不确 定性和缺乏全局测量信息的鲁棒性等优点.

在生物学上，集群所指的是一种智能体依靠局部 感知和相互作用而自主决定运动状态，并由单个智能 体局部行为构成多智能体全局行为的协同方式 ${ }^{[8]}$. 因 此，分布式编队控制的研究更适用于集群系统. 现有 文献中关于集群系统的编队控制方法主要有基于行为 法的编队控制、基于虚拟结构的编队控制、基于人工 势场法的编队控制、基于图论法的编队控制以及基于 领航者-跟随者法的编队控制 ${ }^{[9]}$. 目前, 这几种研究方 法已经逐步地融合在一起，难以绝对地进行区分. 特 别地，基于图论法的编队控制可以借鉴成熟的图论理 论来建立智能体间的感知拓扑图，设计编队控制律， 将代数与几何完美地结合起来, 近年来受到专家学者 的广泛关注，逐步地融合了其他几种方法，成为研究 编队控制的主流方法.

尽管近年来有关集群系统编队控制的研究取得了 不少的成果, 但也存在很多亟需解决的问题 ${ }^{[9]}$, 比如立 体编队控制问题，具有强非线性模型的编队控制问题， 异构集群系统的编队控制问题，通信、感知约束条件 下的编队控制问题，集群系统编队控制系统实例研究 等. 因此, 通过开展对集群系统立体编队控制的研究, 能够为多个智能体在实际中协同执行复杂任务时的编 队提供一定的参考意义.

现有的大多数文献中, 关于集群系统的编队控制 问题提出了一系列的假设或需要一些限制条件：文献 $[10,11]$ 在编队过程中需要用到全局坐标系里的位置信 息; 文献[12,13]中要求智能体期望的航向相同; 文献 $[14,15]$ 中需要智能体间进行内部通信; 文献[16]中假 设智能体间的感知拓扑图为完全图. 此外有一类编队 控制策略不需要这些假设条件, 就能够使智能体自主 地实现所需的编队队形. 文献[17]中给出了一种基于 距离的编队控制策略，文献[18,19]提供了基于方位的 编队控制策略. 文献[20 22]中则研究了基于质心坐标 的编队控制策略，与基于距离以及基于方位编队控制 策略不同的是，在基于质心坐标的编队控制策略中,
智能体的期望队形是通过它们之间的距离以及智能体 与邻居之间所张成的方位角来定义的. 由于常见的传 感器, 如激光扫描仪、雷达、声呐和立体相机, 可以 提供角度和距离的测量, 这项编队策略的重点是编队 队形的结构. 基于图拉普拉斯矩阵的编队控制又可以 归类为一种新型的编队控制策略，编队队形最终能够 收玫至所设计的拉普拉斯矩阵的核空间之内，而不需 要在控制律内引入编队偏置向量, 典型的方法有基于 边拉普拉斯矩阵 ${ }^{[23]}$ 以及基于符号拉普拉斯矩阵 ${ }^{[24,25]}$ 的 编队控制策略，这类策略重视的是特殊的拉普拉斯矩 阵构型所带来的新的性质.

不同于先前解决编队控制问题所涉及的单一研究 方法, 本文旨在结合图论、矩阵论、凸优化理论及李 雅普诺夫稳定性理论中的相关知识, 主要的研究特色 可以归纳如下:

(1) 针对立体空间内运动的二阶动力学模型, 提出 一种基于增益矩阵的集群系统编队控制策略，并利用 半正定规划算法对增益矩阵进行求取;

(2) 设计编队生成和保持控制律使得集群系统在 只需要测量智能体间的相对位置信息、内部不需要通 信的情况下就能够收玫到并保持期望的立体队形;

(3) 提出编队控制律能够在集群系统受到扰动以 及输入饱和限制时, 具备一定的鲁棒性, 并且利用启 发式算法解决编队生成中的避碰问题;

(4) 在编队保持控制策略中, 引入距离约束函数, 保证集群系统编队能够维持期望的固定大小的立体 队形;

(5) 将无领航者的编队控制律扩展到领航者-跟随 者网络下的立体编队控制问题，设计跟随者的分布式 控制律保证集群系统的立体编队的稳定性.

\section{2 问题描述}

\section{1 图论基础}

本文利用图的顶点和边来表示集群系统中的智能 体和它们之间的通讯拓扑关系. 定义一个无向图 $\mathcal{G}=(\mathcal{V}$, $\mathcal{E})$, 其中点集 $\mathcal{V}:=\mathbb{N}_{n}$, 边集 $\mathcal{E} \subseteq\{(i, j): i, j \in \mathcal{V}, i \neq j\}$, 图中的 每个顶点代表一个智能体. 如果顶点 $i \in \mathcal{V}$ 和顶点 $j \in \mathcal{V}$ 之 间存在一条边, 则表示智能体 $i$ 和 $j$ 能够从它们的局部坐 标系里获得彼此的相对位置信息. 这种情况下, 称智能 体 $i$ 和 $j$ 为邻居, 并用集合 $\mathcal{N}_{i}:=\{j \in \mathcal{V}: \mid(i, j) \in \mathcal{E}\}$ 来表示智 
能体 $i$ 的邻居集合. 对于一个顶点序列 $P=v_{1}, v_{2}, \ldots, v_{k}$, 如果每一对相邻的顶点 $v_{i}$ 和 $v_{i+1}$ 之间都存在一条边, 则 称该无向图是连通的.

图 $\mathcal{G}$ 的邻接矩阵 $\mathbf{M}=\left[m_{i j}\right] \in \mathbb{R}^{n \times n}$ 定义如式(1): 当 $(j$, $i) \in \mathcal{E}$ 时, $m_{i j}=1$; 当 $(j, i) \notin \mathcal{E}$ 时, $m_{i j}=0$. 除非特别说明, 否 则不允许有自身边存在 $\left(m_{i i}=0\right)$, 且当图 $\mathcal{G}$ 为无向图时, 对于所有的 $i \neq j$, 有 $m_{i j}=m_{j i}$.

$\mathbf{M}(i, j)= \begin{cases}1, & \text { if }(j, i) \in \mathcal{E}, i \neq j, \\ 0, & \text { if }(j, i) \notin \mathcal{E}, i \neq j, \\ 0, & \text { if } i=j .\end{cases}$

本文中用邻接矩阵 $\mathbf{M}$ 来表示智能体的拓扑结构, 取 $m_{i j}=1$ 时表示智能体 $i$ 和 $j$ 为邻居, $m_{i j}=0$ 时表示智能体 $i$ 和 $j$ 不能获取彼此的信息.

图 $\mathcal{G}$ 的拉普拉斯矩阵一般用 $\underline{\mathbf{L}}=\left[l_{i j}\right] \in \mathbb{R}^{n \times n}$ 表示, 定 义如式(2): 对于 $j \in \mathcal{N}_{i}, l_{i j}=-m_{i j}, i \neq j$; 对于 $j \notin \mathcal{N}_{i}, l_{i j}=0$, $i \neq j$, 对角线元素 $l_{i i}=\sum_{j=1, j \neq i}^{n} m_{i j}$.

$\mathbf{L}(i, j)= \begin{cases}-m_{i j}, & \text { if } i \neq j \text { and } j \in \mathcal{N}_{i}, \\ 0, & \text { if } i \neq j \text { and } j \notin \mathcal{N}_{i}, \\ \sum_{j=1, j \neq i}^{n} m_{i j}, & \text { if } i=j .\end{cases}$

对于一个无向图, 矩阵 $\mathbf{L}$ 是对称半正定的, 并且由 于 $\mathbf{L}$ 的各行元素之和为 0,0 是 $\mathbf{L}$ 的一个特征值, 其对应 的特征向量是元素均为 1 的 $n$ 维列向量 $\mathbf{1}_{n} \in \mathbb{R}^{n}$.

\section{2 问题模型}

考虑包含具有二阶动力学模型的 $n \in \mathbb{N}^{+}$个智能体 的集群系统, 单个智能体 $i$ 的动力学表示为

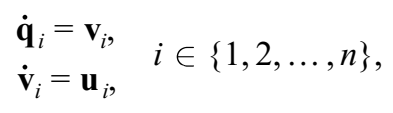

其中, $\mathbf{q}_{i}:=\left[x_{i}, y_{i}, z_{i}\right]^{\mathrm{T}} \in \mathbb{R}^{3}$ 表示智能体 $i$ 在全局坐标系里的 位置, $\mathbf{v}_{i}:=\left[v_{i x}, v_{i y}, v_{i z}\right]^{\mathrm{T}} \in \mathbb{R}^{3}$ 表示智能体 $i$ 的速度, $\mathbf{u}_{i} \in \mathbb{R}^{3}$ 则 表示智能体 $i$ 的控制输入.

令 $\mathbf{q}:=\left[\mathbf{q}_{1}{ }^{\mathrm{T}}, \mathbf{q}_{2}{ }^{\mathrm{T}}, \ldots, \mathbf{q}_{n}{ }^{\mathrm{T}}\right]^{\mathrm{T}} \in \mathbb{R}^{3 n}, \mathbf{v}:=\left[\mathbf{v}_{1}{ }^{\mathrm{T}}, \mathbf{v}_{2}{ }^{\mathrm{T}}, \ldots, \mathbf{v}_{n}{ }^{\mathrm{T}}\right]^{\mathrm{T}}$ $\in \mathbb{R}^{3 n}, \mathbf{u}:=\left[\mathbf{u}_{1}{ }^{\mathrm{T}}, \mathbf{u}_{2}{ }^{\mathrm{T}}, \ldots, \mathbf{u}_{n}{ }^{\mathrm{T}}\right]^{\mathrm{T}} \in \mathbb{R}^{3 n}$ 分别表示系统的聚合 位置向量、聚合速度向量以及聚合控制向量, 则整个
系统的动力学方程可以表示为

$\dot{\mathbf{q}}=\mathbf{v}$,

$\dot{\mathbf{v}}=\mathbf{u}$,

$i \in\{1,2, \ldots, n\}$.

本文的主要目标是针对式(4)所示的二阶集群系 统, 设计相应的分布式控制律, 使得每个智能体在只需 获得其邻居智能体相对位置信息的情况下, 构造出增 益矩阵，并保证整个编队收敛到期望的立体队形，同 时考虑避碰、队形的保持控制等问题, 最后将所设计 的控制律进行改进, 应用到领航者-跟随者网络下的编 队控制, 使得跟随者跟随领航者形成期望的立体队形. 本文中假设智能体间的感知拓扑为无向刚性图, 期望 的立体队形是物理可行的.

\section{3 二阶集群系统立体编队生成控制}

\section{1 控制律设计}

为了使智能体收玫到期望的立体队形, 智能体 $i$ 的 控制律设计为

$\mathbf{u}_{i}=k_{0} \sum_{j \in \mathcal{N}_{i}} \mathbf{A}_{i j}\left(\mathbf{q}_{j}-\mathbf{q}_{i}\right)-k_{1} \mathbf{v}_{i}$,

其中, $\mathbf{A}_{i j} \in \mathbb{R}^{3 \times 3}$ 为待求的增益矩阵, $\mathbf{q}_{j}-\mathbf{q}_{i}$ 表示智能体 $j$ 在 智能体 $i$ 局部坐标系里的位置, $k_{0}, k_{1} \in \mathbb{R}$ 为常量控制增 益. 给定增益矩阵的形式:

$\mathbf{A}_{i j}=\left[\begin{array}{ccc}a_{i j} & -b_{i j} & 0 \\ b_{i j} & a_{i j} & 0 \\ 0 & 0 & c_{i j}\end{array}\right], a_{i j}, b_{i j}, c_{i j} \in \mathbb{R}$.

假设智能体局部坐标系的 $z$ 轴和全局坐标系的 $z$ 轴 对齐, 由 $\mathbf{A}_{i j}$ 的可交换性, 可知用智能体间局部坐标系 下的 $\mathbf{q}_{i}$ 和 $\mathbf{q}_{j}$ 表示的闭环动力学方程和全局坐标表示的 相同, 并且局部坐标系并不需要沿着 $x-y$ 平面方向对 齐 $^{[26]}$. 因此, 所设计的控制律是分布式的, 编队过程 中, 每个智能体只需要知道其邻居智能体的相对位置 信息.

则系统的闭环动力学方程可以表示为

$\left[\begin{array}{c}\dot{\mathbf{q}} \\ \dot{\mathbf{v}}\end{array}\right]=\underbrace{\left[\begin{array}{cc}\mathbf{0} & \mathbf{I}_{3 n} \\ k_{0} \mathbf{A} & -k_{1} \mathbf{I}_{3 n}\end{array}\right]}_{\overline{\mathbf{A}}}\left[\begin{array}{l}\mathbf{q} \\ \mathbf{v}\end{array}\right]$, 
$\mathbf{A}=\left[\begin{array}{cccc}-\sum_{j=2}^{n} \mathbf{A}_{1 j} & \mathbf{A}_{12} & \cdots & \mathbf{A}_{1 n} \\ \mathbf{A}_{21} & -\sum_{j=1}^{n} \mathbf{A}_{2 j} & \cdots & \mathbf{A}_{2 n} \\ \vdots & & \ddots & \vdots \\ \mathbf{A}_{n 1} & \mathbf{A}_{n 2} & \cdots & -\sum_{j=1}^{n-1} \mathbf{A}_{n j}\end{array}\right] \in \mathbb{R}^{3 n \times 3 n}$

其中, 对于 $j \notin \mathcal{N}_{i}, \mathbf{A}_{i j}=0$. 注意到矩阵 $\mathbf{A}$ 的 $3 \times 3$ 对角块是 同一行其余块的负和, 矩阵 $\mathbf{A}$ 为块拉普拉斯结构. 因而, 向量

$\mathbf{1}_{x}=[1,0,0,1,0,0, \ldots, 1,0,0]^{\mathrm{T}} \in \mathbb{R}^{3 n}$,

$\mathbf{1}_{y}=[0,1,0,0,1,0, \ldots, 0,1,0]^{\mathrm{T}} \in \mathbb{R}^{3 n}$,

$\mathbf{1}_{z}=[0,0,1,0,0,1, \ldots, 0,0,1]^{\mathrm{T}} \in \mathbb{R}^{3 n}$

在 $\mathbf{A}$ 的核空间内.

\section{2 稳定性证明}

引理 $\mathbf{1}^{[26]}$ 假设矩阵 $\mathrm{A}$ 的所有非零特征根均具有负 实部, 那么系统 $\dot{\mathbf{q}}=\mathbf{A q}$ 的所有运动轨迹指数收玫到 $\mathbf{A}$ 的核.

令 $\mathbf{q}^{*} \in \mathbb{R}^{3 n}$ 表示智能体期望队形的位置向量, $\mathbf{q}^{*} \in$ $\mathbb{R}^{3 n}$ 表示期望队形的位置向量绕 $z$ 轴旋转 $90^{\circ}$ 得到的位置 向量, $\overline{\mathbf{q}}_{i}^{*} \in \mathbb{R}^{3 n}$ 表示期望的位置向量在 $x-y$ 平面上的投影. 即

$$
\begin{aligned}
& \mathbf{q}^{*}=\left[\mathbf{q}_{1}^{* \mathrm{~T}}, \mathbf{q}_{2}^{* \mathrm{~T}}, \ldots, \mathbf{q}_{n}^{* \mathrm{~T}}\right]^{\mathrm{T}} \in \mathbb{R}^{3 n}, \\
& \overline{\mathbf{q}}^{*}=\left[\overline{\mathbf{q}}_{1}^{* \mathrm{~T}}, \overline{\mathbf{q}}_{2}^{* \mathrm{~T}}, \ldots, \overline{\mathbf{q}}_{n}^{* \mathrm{~T}}\right]^{\mathrm{T}} \in \mathbb{R}^{3 n}, \\
& \overline{\mathbf{q}}^{*}=\left[\overline{\mathbf{q}}_{1}^{* \mathrm{~T}}, \overline{\overline{\mathbf{q}}}_{2}^{* \mathrm{~T}}, \ldots, \overline{\mathbf{q}}_{n}^{* \mathrm{~T}}\right]^{\mathrm{T}} \in \mathbb{R}^{3 n} .
\end{aligned}
$$

则有如下定理.

定理1 对于具有二阶动力学式(3)和控制律式(5) 的三维集群系统, 如果满足以下两个条件:

(1) 向量 $\mathbf{1}_{x}, \mathbf{1}_{y}, \mathbf{1}_{z}, \mathbf{q}^{*}, \mathbf{q}^{*}, \overline{\mathbf{q}}_{i}^{*}$ 构成 $\mathbf{A}$ 的核空间的一 组基;

(2) 系统矩阵 $\mathbf{A}$ 的非零特征根, 即对于 $\mathbf{A}$ 的所有特 征值 $\mu \in \sigma(\mathbf{A})$, 二次方程 $\lambda^{2}+k_{1} \lambda-k_{0} \mu=0$ 的所有非零根均 具有负实部.

则智能体全局收敛到期望的立体队形(包含绕 $z$ 轴 的旋转、平移，沿着 $z$ 轴方向的缩放以及沿着 $x-y$ 平面 方向的缩放).
证明 由条件(2)可知系统矩阵 $\mathbf{A}$ 的非零特征根均 具有负实部, 系统矩阵 $\mathbf{A}$ 为稳定的状态矩阵, 由引理 1 可知系统的状态向量 $\left[\mathbf{q}^{\mathrm{T}}, \mathbf{v}^{\mathrm{T}}\right]^{\mathrm{T}}$ 能够收玫到系统矩阵 $\mathbf{A}$ 的核. 即

$\left[\begin{array}{cc}\mathbf{0} & \mathbf{I}_{3 n} \\ k_{0} \mathbf{A} & -k_{1} \mathbf{I}_{3 n}\end{array}\right]\left[\begin{array}{l}\mathbf{q} \\ \mathbf{v}\end{array}\right]=\mathbf{0}$

$$
\left\{\begin{array}{c}
\mathbf{v}=\mathbf{0} \\
\mathbf{A q}=\mathbf{0}
\end{array}\right.
$$

因而, q收玫到 $\mathbf{A}$ 的核空间，而 $\mathbf{A}$ 的核空间即期望的 位置向量关于 $z$ 轴的旋转、平移，沿着 $z$ 轴方向的缩放 以及沿着 $x-y$ 平面的缩放等, 即智能体全局收玫到期望 的立体队形.

注1 文献[20, 定理3.2]中研究了满足定理1中条件 的对称矩阵 $\mathbf{A}$ 存在的拓扑条件：感知拓扑为无向刚性 图. 本文中假设给定的所有感知拓扑都满足这个条件.

\section{3 增益矩阵 $\mathrm{A}$ 的优化求解}

针对具有刚性通讯拓扑的集群系统立体编队控 制，Fathian等人在文献[26]中给出了二维空间内的集 群系统增益矩阵 $\mathbf{A}$ 的优化求解算法, 如表1所示, 本文 将其改进并应用到三维空间, 该方法能够极大化系统 对扰动、测量误差等的鲁棒性.

在后续的仿真实验中, 可以使用MATLAB中的 $\mathrm{CVX}^{[27]}$ 工具箱来求解满足条件的稳定的增益矩阵 $\mathbf{A}$.

注2 由于求出的增益矩阵 $\mathbf{A}$ 为对称矩阵, 因此在 实际设计控制律时, 只需选取控制增益 $k_{0}, k_{1}>0$ 即可. 在接下来的性质分析中, 均假设控制增益 $k_{0}, k_{1}>0$.

\section{4 鲁棒性分析}

\subsection{1 系统对扰动的鲁棒性}

引理 2 令 $\mathbf{R} \in \mathrm{SO}(3)$ 表示关于单位正规旋转坐标系 $[x, y, z]^{\mathrm{T}} \in \mathbb{R}^{3}$ 旋转 $\theta \in[-\pi, \pi)$ 弧度角的旋转矩阵. 如 果 $|\theta|<\frac{\pi}{2}$, 则矩阵 $\mathbf{R}+\mathbf{R}^{\mathrm{T}}$ 为正定矩阵.

证明 矩阵 $\mathbf{R}$ 可以表示成

$\mathbf{R}=\left[\begin{array}{ccc}c+x^{2} \alpha & x y \alpha-z s & x z \alpha+y s \\ y x \alpha+z s & c+y^{2} \alpha & y z \alpha-x s \\ z x \alpha-y s & z y \alpha+x s & c+z^{2} \alpha\end{array}\right]$, 
表 1 增益矩阵 $\mathbf{A}$ 的优化求解算法

Table 1 Algorithm for optimal calculation of gain matrix $\mathbf{A}$

\begin{tabular}{|c|c|}
\hline 算法1: 增益矩阵 $\mathbf{A}$ 的优化求解 & \\
\hline $\begin{array}{l}\text { 输入: 期望队形坐标 } \mathbf{q}^{*} \in \mathbb{R}^{3 n} . \\
\text { 输出: 增益矩阵 } \mathbf{A} \in \mathbb{R}^{3 n \times 3 n} . \\
\text { 步骤1: 令 } \mathbf{N}=\left[\mathbf{q}^{*}, \mathbf{q}^{*}, \overline{\mathbf{q}}^{*}, \mathbf{1}_{x}, \mathbf{1}_{y}, \mathbf{1}_{z}\right] . \\
\text { 步骤2: 对 } \mathbf{N} \text { 进行奇异值分解, 得到 } \mathbf{N}=\mathbf{U S V}^{\mathrm{T}} \text {. } \\
\text { 步骤3: 令 } \mathbf{Q} \text { 为 } \mathbf{U} \text { 的后 } 3 n-6 \text { 列向量组成的矩阵. }\end{array}$ & \\
\hline $\begin{array}{l}\text { 步骤4: 使用SDP求解器求解式 } \begin{array}{c}\mathbf{A}=\underset{a_{i j}}{\operatorname{argmax}}, b_{i j}, c_{i j} \\
\text { subject to }\end{array} \\
\text { 正定规划问题. }\end{array}$ & $\begin{array}{l}\lambda_{\text {min }}(-\overline{\mathbf{A}}) \\
\mathbf{A N}=0\end{array}$ \\
\hline
\end{tabular}

其中, $\alpha:=1-c, c$ 和 $s$ 分别为 $\cos \theta, \sin \theta$ 的简写. 因此,

$\mathbf{R}+\mathbf{R}^{\mathrm{T}}=2 \alpha\left[\begin{array}{ccc}\beta+x^{2} & x y & x z \\ y x & \beta+y^{2} & y z \\ z x & z y & \beta+z^{2}\end{array}\right]$,

其中, $\beta:=\frac{c}{1-c}$. 注意到 $x^{2}+y^{2}+z^{2}=1$, 通过直接计算可以 得到 $\mathbf{R}+\mathbf{R}^{\mathrm{T}}$ 的特征值为 $\{2 \alpha, 2 \alpha, 2 \alpha \beta\}$. 由 $|\theta|<\frac{\pi}{2}$, 可知 $\alpha$, $\beta>0$, 从而矩阵 $\mathbf{R}+\mathbf{R}^{\mathrm{T}}$ 为正定矩阵.

所设计的控制律对扰动具有较好的鲁棒性.

定理 2 考虑满足定理 1条件的增益矩阵 $\mathbf{A}$, 令 $\mathbf{R}_{i} \in \mathrm{SO}(3)$ 表示关于任意坐标轴旋转 $\theta_{i}$ 弧度角的旋转矩 阵, $c_{i} \in \mathbb{R}^{+}$. 如果对任意小的 $\varepsilon>0$, 有 $c_{i} \geq \varepsilon$, $\theta_{i} \in\left[-\frac{\pi}{2}+\varepsilon, \frac{\pi}{2}-\varepsilon\right]$, 则存在扰动控制:

$\mathbf{u}_{i}=c_{i} \mathbf{R}\left[k_{0} \sum_{j \in \mathcal{N}_{i}} \mathbf{A}_{i j}\left(\mathbf{q}_{j}-\mathbf{q}_{i}\right)-k_{1} \mathbf{v}_{i}\right]$.

因而, 智能体全局收玫到期望的立体队形(包含关 于 $z$ 轴的旋转、平移, 沿着 $z$ 轴方向的缩放以及沿着 $x-y$ 平面的缩放).

证明 旋转矩阵 $\mathbf{R}_{i}$ 可以表示成

$$
\begin{aligned}
& \mathbf{R}_{i}=\left[\begin{array}{ccc}
c_{i}+x_{i}{ }^{2} \alpha_{i} & x_{i} y_{i} \alpha_{i}-z_{i} s_{i} & x_{i} z_{i} \alpha_{i}+y_{i} s_{i} \\
y_{i} x_{i} \alpha_{i}+z_{i} s_{i} & c_{i}+y_{i}{ }^{2} \alpha_{i} & y_{i} z_{i} \alpha_{i}-x_{i} s_{i} \\
z_{i} x_{i} \alpha_{i}-y_{i} s_{i} & z_{i} y_{i} \alpha_{i}+x_{i} s_{i} & c_{i}+z_{i}{ }^{2} \alpha_{i}
\end{array}\right], \\
& \theta_{i} \in\left(-\frac{\pi}{2}, \frac{\pi}{2}\right),
\end{aligned}
$$

其中, $x_{i}=\frac{u_{i x}}{\left\|\mathbf{u}_{i}\right\|}, y_{i}=\frac{u_{i y}}{\left\|\mathbf{u}_{i}\right\|}, z_{i}=\frac{u_{i z}}{\left\|\mathbf{u}_{i}\right\|}$,

$\alpha_{i}:=1-c_{i}$, 而 $c_{i}, s_{i}$ 分别为 $\cos \theta_{i}, \sin \theta_{i}$ 的简写.

加入扰动后系统的控制律:

$\mathbf{u}=\mathbf{C R}\left(k_{0} \mathbf{A q}-k_{1} \mathbf{v}\right)$,

其中, $\mathbf{C}=\operatorname{diag}\left(c_{1} \mathbf{I}_{3}, c_{2} \mathbf{I}_{3}, \ldots, c_{n} \mathbf{I}_{3}\right) \in \mathbb{R}^{3 n \times 3 n}, \mathbf{R}=\operatorname{diag}\left(\mathbf{R}_{1}, \mathbf{R}_{2}\right.$, $\left.\ldots, \mathbf{R}_{n}\right) \in \mathbb{R}^{3 n \times 3 n}$ 为块对角矩阵. 则系统的闭环动力学方 程可以写成

$$
\begin{aligned}
\dot{\mathbf{q}} & =\mathbf{v}, \\
\dot{\mathbf{v}} & =\mathbf{C R}\left(k_{0} \mathbf{A q}-k_{1} \mathbf{v}\right) .
\end{aligned}
$$

定义一个备选李雅普诺夫函数:

$$
V_{1}=-k_{0} \mathbf{q}^{\mathrm{T}} \mathbf{A} \mathbf{q}+\frac{1}{2} \mathbf{v}^{\mathrm{T}} \mathbf{C}^{-1}\left(\mathbf{R}+\mathbf{R}^{\mathrm{T}}\right) \mathbf{v} .
$$

由 $\mathbf{A}$ 为半负定矩阵, 及引理 2 可知 $V_{1} \geq 0$, 注意到 $\mathbf{A}=\mathbf{A}^{\mathrm{T}}, \mathbf{R} \mathbf{R}^{\mathrm{T}}=\mathbf{I}_{3 n}$, 则 $V_{2}$ 沿着系统式(19)运动轨迹的导数为

$$
\begin{aligned}
\dot{V}_{1}= & -2 k_{0} \mathbf{q}^{\mathrm{T}} \mathbf{A} \mathbf{v}+\dot{\mathbf{v}}^{\mathrm{T}} \mathbf{C}^{-1} \mathbf{R} \mathbf{v}+\mathbf{v}^{\mathrm{T}} \mathbf{C}^{-1} \mathbf{R}^{\mathrm{T}} \dot{\mathbf{v}} \\
= & -2 k_{0} \mathbf{q}^{\mathrm{T}} \mathbf{A} \mathbf{v}+\left(k_{0} \mathbf{q}^{\mathrm{T}} \mathbf{A} \mathbf{R}^{\mathrm{T}} \mathbf{C}-k_{1} \mathbf{v}^{\mathrm{T}} \mathbf{R}^{\mathrm{T}} \mathbf{C}\right) \mathbf{C}^{-1} \mathbf{R} \mathbf{v} \\
& +\mathbf{v}^{\mathrm{T}} \mathbf{C}^{-1} \mathbf{R}^{\mathrm{T}}\left(k_{0} \mathbf{C} \mathbf{R} \mathbf{A q}-k_{1} \mathbf{C R v}\right) \\
= & -2 k_{0} \mathbf{q}^{\mathrm{T}} \mathbf{A} \mathbf{v}+2 k_{0} \mathbf{q}^{\mathrm{T}} \mathbf{A} \mathbf{v}-2 k_{1} \mathbf{v}^{\mathrm{T}} \mathbf{v} \\
= & -2 k_{1} \mathbf{v}^{\mathrm{T}} \mathbf{v} \leq 0 .
\end{aligned}
$$

从而可知系统是稳定的, 由 $\dot{V}_{1}=0$, 可知 $\mathbf{v}=\mathbf{0}$, 又 由 $\dot{\mathbf{v}}=\mathbf{0}$, 可知 $\mathbf{R A q}=\mathbf{0}$. 而矩阵 $\mathbf{R}$ 列满秩, 从而可知 $\mathbf{A q}=\mathbf{0}$. 由李雅普诺夫稳定性理论及拉萨尔不变性定理 可知, $\mathbf{q}$ 收玫到最大不变集 $\left\{\mathbf{q} \in \mathbb{R}^{3 n} \mid \mathbf{A q} \equiv 0\right\}$, 即 $\mathbf{A}$ 的核 空间, 从而可知智能体能够收玫到期望的立体队形.

由定理 2 可知, 所设计的控制律对扰动具有较好的 鲁棒性. 因此, 可以在编队策略中加入一个启发式的旋 转智能体 $i$ 的控制向量 $\mathbf{u}_{i}$ 的避碰算法. 图1描述了当智能 体1可能会与邻居智能体发生碰撞时, 通过旋转控制向 量 $\mathbf{u}_{1}$ 使其不与预先定义的碰撞区域相交, 即可避免发 生碰撞. 由于控制向量 $\mathbf{u}_{1}$ 与智能体 3 的安全区域有交 集, 为了达到避碰, 智能体 1 需要向包含以 $\mathbf{u}_{1}$ 作为法向 量的平面(图1中通过智能体 1 的灰色平面)的上半开空 间的任意方向移动, 而表2中给出了相应的避碰算法.

\subsection{2 系统对输入饱和的鲁棒性}

实际上一个智能体的加速度不可能为任意大的数 


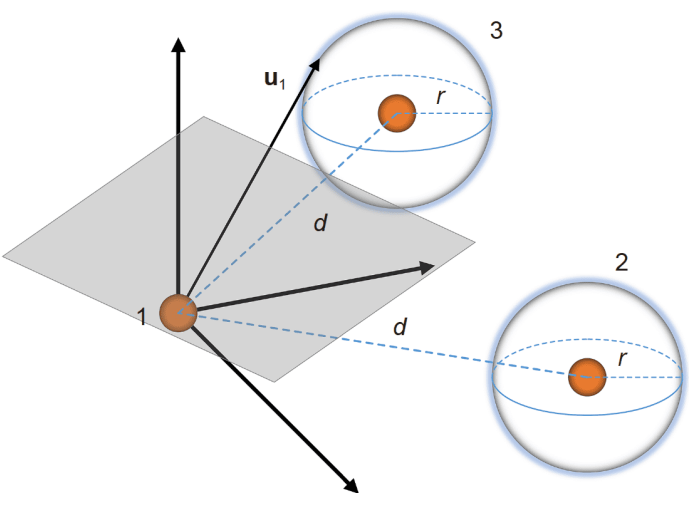

图 1 (网络版彩图)旋转智能体 1 的控制向量 $\mathbf{u}_{1}$ 避免与智能 体3的碰撞

Figure 1 (Color online) Rotating the control vector $\mathbf{u}_{1}$ of the agent 1 to avoid collision between the agent 3 .

表 2 避碰算法

Table 2 Algorithm for collision avoidance

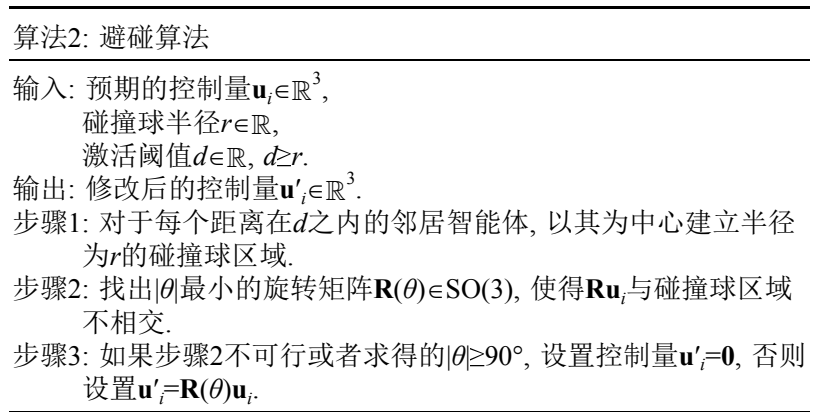

值, 因而智能体的控制输入有一个饱和值, 但这并不会 影响智能体收敛到期望的立体队形.

引理 $\mathbf{3}^{[26]}$ 一组切换动力系统 $\dot{\mathbf{x}}=f_{i}(\mathbf{x}), i=1,2, \ldots, N$. 令 $V: \mathbb{R}^{n} \rightarrow \mathbb{R}$ 表示一个正定, 连续可微, 且径向有界的函 数. 如果有 $\frac{\partial V}{\partial \mathbf{x}} f_{i}(\mathbf{x})<0, \forall \mathbf{x} \neq \mathbf{0}, \forall i$, 则此切换系统全局 一致渐近稳定.

推论 $\mathbf{1}^{[28]}$ 引理 3 可以拓展到一个具有零 集 $Z:=\left\{\mathbf{x} \in \mathbb{R}^{n}: V(\mathbf{x})=0\right\}$ 的半正定的函数 $V$, 在这种 情况下, 如果有 $\frac{\partial V}{\partial \mathbf{x}} f_{i}(\mathbf{x})<0, \forall \mathbf{x} \neq 0, \forall i$, 则系统的所有 运动轨迹全局一致渐近收玫到 $Z$.

定理3 考虑具有二阶动力学式(3)的集群系统, 同 时假定 $u_{\text {max }}>0$ 为一个正实数. 如果 $\mathbf{u}_{i}$ 会达到饱和, 即 $|\mathbf{u}|_{i} \leq$ $u_{\text {max }}$, 则在控制律式(5)下, 集群系统依然能够收玫到期 望的立体队形.
证明 为了建立输入饱和的模型, 定义对角矩阵 $\mathbf{S}=\operatorname{diag}\left(s_{11} \mathbf{I}_{3}, s_{22} \mathbf{I}_{3}, \ldots, s_{n n} \mathbf{I}_{3}\right) \in \mathbb{R}^{3 n \times 3 n}$, 其对角线元素为

$$
s_{i i}=\left\{\begin{array}{cc}
1, & \text { if }\left|\mathbf{u}_{i}\right| \leq u_{\max }, \\
\frac{u_{\max }}{\left|\mathbf{u}_{i}\right|}, & \text { if }\left|\mathbf{u}_{i}\right|>u_{\text {max }} .
\end{array}\right.
$$

如图2所示， $\mathbf{S}$ 的对角线元素可以看成关于控制输 入 $\mathbf{u}_{i}$ 的饱和函数.

从而具有饱和输入的闭环动力学方程为

$$
\begin{aligned}
\dot{\mathbf{q}} & =\mathbf{v}, \\
\dot{\mathbf{v}} & =\mathbf{S}\left(k_{0} \mathbf{A q}-k_{1} \mathbf{v}\right) .
\end{aligned}
$$

系统式(23)可以理解为一组切换动力系统，其解 在Filippov意义上有明确的定义. 为了便于分析, 当控 制输入量的模值 $\left|\mathbf{u}_{i}\right|>u_{\text {max }}$ 时, 相当于将输入饱和建模成 输入量 $\mathbf{u}_{i}$ 乘以一个正的比例缩小因子 $s_{i i}$, 且满足 $0<$ $s_{i i}<1$, 智能体 $i$ 实际的控制输入变为 $s_{i i} \mathbf{u}_{i}$. 从而由引理 3、推论 1 及定理 2 可知, 输入饱和并不会影响集群系 统收敛到期望的队形.

\section{4 二阶集群系统立体编队保持控制}

\section{1 控制律设计}

在编队生成期望的立体队形后，进一步为了保持 队形大小的固定，可以使用下面的控制律:

$\mathbf{u}_{i}=k_{0} \sum_{j \in \mathcal{N}_{i}} \mathbf{A}_{i j}\left(\mathbf{q}_{j}-\mathbf{q}_{i}\right)+\varepsilon * f\left(d_{i j}-d_{i j}^{*}\right)\left(\mathbf{q}_{j}-\mathbf{q}_{i}\right)-k_{1} \mathbf{v}_{i}$,

其中, $\mathbf{A}_{i j}$ 的定义和式(6)相同, $d_{i j}=\left\|\mathbf{q}_{j}-\mathbf{q}_{i}\right\|, d_{i j}{ }^{*}=\left\|\mathbf{q}_{j}{ }^{*}-\mathbf{q}_{i}{ }^{*}\right\|$, $\varepsilon>0$ 为增益, $f: \mathbb{R} \rightarrow \mathbb{R}$, 为有界函数, 且对于 $x \neq 0, x f(x)>0, f(0)$ $=0$. 可以选择 $f: x \mapsto \frac{1}{k} \arctan (x)$ 或者 $f: x \mapsto \frac{1}{k} \tanh (x)$, 其中 $k>0$ 为任意常量. 函数 $f$ 的作用是当邻居智能体的 距离大于期望值时，将其距离拉近，小于时则使其远 离. 从而可以得到系统的的闭环动力学方程:

$\left[\begin{array}{l}\dot{\mathbf{q}} \\ \dot{\mathbf{v}}\end{array}\right]=\left[\begin{array}{cc}0 & \mathbf{I}_{3 n} \\ k_{0} \mathbf{A}+\varepsilon \mathbf{F} & -k_{1} \mathbf{I}_{3 n}\end{array}\right]\left[\begin{array}{l}\mathbf{q} \\ \mathbf{v}\end{array}\right]$,

其中，增益矩阵 $\mathbf{A}$ 可以利用算法1求解得到, $F(q): \mathbb{R}^{3 n}$ $\rightarrow \mathbb{R}^{3 n}$ 的定义为 

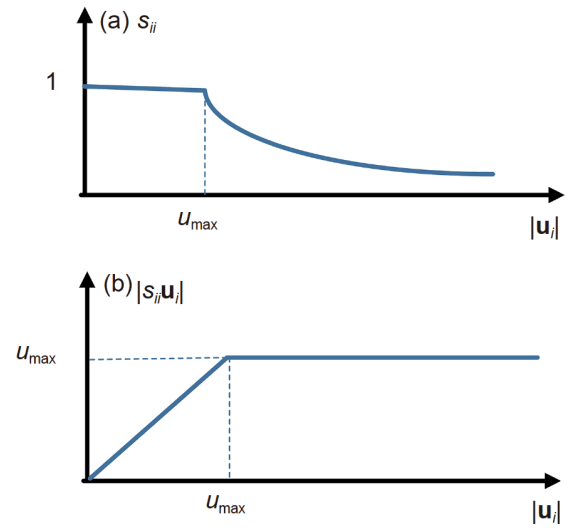

图 2 (网络版彩图)输入饱和鲁棒性分析曲线. (a) 矩阵 $\mathbf{S}$ 对 角线上的第 $i$ 个元素与控制输入关系曲线; (b) 输入饱和对控 制输入的影响曲线

Figure 2 (Color online) Analysis curves for robustness of input saturations. (a) Relationship curve between the $i$ th element in diagonal of matrix $\mathbf{S}$ and control inputs; (b) relationship curve between saturations and control inputs.

$\mathbf{F}(\mathbf{q})=\left[\begin{array}{cccc}-\sum_{j \neq 1} \mathbf{F}_{1 j} & \mathbf{F}_{12} & \cdots & \mathbf{F}_{1 n} \\ \mathbf{F}_{21} & -\sum_{j \neq 2} \mathbf{F}_{2 j} & \cdots & \mathbf{F}_{2 n} \\ \vdots & & \ddots & \vdots \\ \mathbf{F}_{n 1} & \mathbf{F}_{n 2} & \cdots & \mathbf{F}_{n n}\end{array}\right]$.

$3 \times 3$ 的非对角块元素定义为

$\mathbf{F}_{i j}= \begin{cases}f\left(d_{i j}-d_{i j}^{*}\right) \mathbf{I}_{3}, & \text { if } j \in \mathcal{N}_{i}, \\ 0, & \text { otherwise. }\end{cases}$

\section{2 稳定性证明}

引理 $4^{[29]}$ 考虑奇异摄动系统:

$\dot{\mathbf{x}}=f(t, \mathbf{x}, \mathbf{z}, \varepsilon)$,

$\varepsilon \dot{\mathbf{z}}=g(t, \mathbf{x}, \mathbf{z}, \varepsilon)$,

其中, $\mathbf{x} \in B^{r} \subset \mathbb{R}^{n}, B^{r}$ 代表关于原点半径为 $r$ 的球, $\mathbf{z} \in \mathbb{R}^{m}$. 如果对于所有的 $(t, \mathbf{x}, \varepsilon) \in[0, \infty) \times B^{r} \times\left[0, \varepsilon_{0}\right]$, 以下几个条 件均满足:

(1) $f(t, 0,0, \varepsilon)=0$, 且 $g(t, 0,0, \varepsilon)=0$;

(2) 方程 $g(t, \mathbf{x}, \mathbf{z}, \varepsilon)=0$ 有一个孤立根 $\mathbf{z}=h(t, \mathbf{x})$, 并且 $h(t, 0)=0$;

(3) 对于 $\mathbf{z}-h(t, \mathbf{x}) \in B_{\rho} \subset \mathbb{R}^{m}$, 函数 $f, g, h$ 以及它们的 二阶偏导数均有界;

(4) 退化系统 $\dot{\mathbf{x}}=f(t, \mathbf{x}, h(t, \mathbf{x}), 0)$ 的原点是指数稳 定的;
(5) 边界层系统 $\frac{\mathrm{d} y}{\mathrm{~d} \tau}=g(t, \mathbf{x}, y+h(t, \mathbf{x}), 0), \tau=\varepsilon t$ 的原 点是指数稳定的, 和 $(t, x)$ 一致.

则存在 $\varepsilon^{*}>0$, 对于所有的 $\varepsilon^{<} \varepsilon^{*}$, 系统式(28)的原点 是指数稳定的.

定理4 考虑具有动力学式(3)和控制律式(24)的二 阶集群系统，如果设计的增益矩阵 $\mathbf{A}$ 对称且满足定理 1 中的条件, 则存在 $\varepsilon^{*}>0$, 使得对于所有的 $0<\varepsilon<\varepsilon^{*}$, 则期望 的队形(包含绕 $z$ 轴的旋转、平移)是一个几乎全局指 数稳定的平衡点.

证明 令 $\mathbf{N}=\left[\mathbf{q}^{*}, \overline{\mathbf{q}}^{*}, \overline{\mathbf{q}}^{*}, \mathbf{1}_{x}, \mathbf{1}_{y}, \mathbf{1}_{z}\right] \in \mathbb{R}^{3 n \times 6}$ 为矩阵 $\mathbf{A}$ 的核空间里的一组基底, 并对矩阵 $\mathbf{N}$ 进行奇异值分解, 得到 $\mathbf{U S V} \mathbf{T}^{\mathrm{T}}=\mathbf{N}$, 其中,

$\mathbf{U}=[\overline{\mathbf{Q}}, \mathbf{Q}] \in \mathbb{R}^{3 n \times 3 n}$.

矩阵 $\mathbf{Q} \in \mathbb{R}^{3 n \times 6}$ 中的每一列表示矩阵 $\mathbf{A}$ 的核空间里 的一组正交向量, 特别地, $\overline{\mathbf{Q}}$ 可以取为 $\overline{\mathbf{Q}}=\left[\overline{\mathbf{Q}}_{1}, \overline{\mathbf{Q}}_{2}\right]$,

$\overline{\mathbf{Q}}_{1}:=\left[\frac{\mathbf{q}^{*}}{\left\|\mathbf{q}^{*}\right\|}, \frac{\mathbf{q}^{*}}{\left\|\mathbf{q}^{*}\right\|}, \frac{\overline{\mathbf{q}}^{*}}{\left\|\overline{\mathbf{q}}^{*}\right\|}\right] \in \mathbb{R}^{3 n \times 3}$,

$\overline{\mathbf{Q}}_{2}=\left[\mathbf{v}_{1}, \mathbf{v}_{2}, \mathbf{v}_{3}\right] \in \mathbb{R}^{3 n \times 3}$,

其中, $\mathbf{v}_{1}, \mathbf{v}_{2}, \mathbf{v}_{3} \in \mathbb{R}^{3 n}$ 是 $\mathbf{1}_{x}, \mathbf{1}_{y}, \mathbf{1}_{z}$ 张成的向量空间里相互 正交的单位向量, 并且都和 $\overline{\mathbf{Q}}_{1}$ 正交.

矩阵 $\mathbf{Q} \in \mathbb{R}^{3 n \times(3 n-6)}$ 由矩阵 $\mathbf{U}$ 的后 $3 n-6$ 列组成, 其中 每一列为矩阵 $\mathbf{Q}$ 的正交补空间里的一组正交向量. 考 虑坐标变换: $\mathbf{p}=\mathbf{U}^{\mathrm{T}} \mathbf{q}$, 令 $\mathbf{p}=\left[\mathbf{p}_{1}{ }^{\mathrm{T}}, \mathbf{p}_{2}{ }^{\mathrm{T}}\right]^{\mathrm{T}}$, 其中 $\mathbf{p}_{1} \in \mathbb{R}^{6}{ }^{6}$ 表示 $\mathbf{p}$ 的前 6 个元素, $\mathbf{p}_{2} \in \mathbb{R}^{3 n-6}$ 表示 $\mathbf{p}$ 的其余元素. 则在新坐标 系下, 得到的闭环动力学方程为

$$
\begin{aligned}
{\left[\begin{array}{l}
\ddot{\mathbf{p}}_{1} \\
\ddot{\mathbf{p}}_{2}
\end{array}\right]=} & k_{0} \mathbf{U}^{\mathrm{T}} \mathbf{A} \mathbf{U}\left[\begin{array}{l}
\mathbf{p}_{1} \\
\mathbf{p}_{2}
\end{array}\right] \\
& +\varepsilon \mathbf{U}^{\mathrm{T}} \mathbf{F U}\left[\begin{array}{l}
\mathbf{p}_{1} \\
\mathbf{p}_{2}
\end{array}\right]-k_{1}\left[\begin{array}{l}
\dot{\mathbf{p}}_{1} \\
\dot{\mathbf{p}}_{2}
\end{array}\right] \\
= & k_{0}\left[\begin{array}{cc}
0 & 0 \\
0 & \mathbf{Q}^{\mathrm{T}} \mathbf{A} \mathbf{Q}
\end{array}\right]\left[\begin{array}{l}
\mathbf{p}_{1} \\
\mathbf{p}_{2}
\end{array}\right] \\
& +\varepsilon\left[\begin{array}{ll}
\overline{\mathbf{Q}}^{\mathrm{T}} \mathbf{F} \overline{\mathbf{Q}} & \overline{\mathbf{Q}}^{\mathrm{T}} \mathbf{F Q} \\
\mathbf{Q}^{\mathrm{T}} \mathbf{F} \mathbf{\mathbf { Q }} & \mathbf{Q}^{\mathrm{T}} \mathbf{F Q}
\end{array}\right]\left[\begin{array}{l}
\mathbf{p}_{1} \\
\mathbf{p}_{2}
\end{array}\right]-k_{1}\left[\begin{array}{l}
\dot{\mathbf{p}}_{1} \\
\dot{\mathbf{p}}_{2}
\end{array}\right],
\end{aligned}
$$

其中, $\mathbf{F}$ 为 $\mathbf{F}(\mathbf{q})$ 的简写. 由于 $\mathbf{F}$ 具有块拉普拉斯结构, 故 $\mathbf{F} 1_{x}=\mathbf{F 1} 1_{y}=\mathbf{F 1} 1_{z}=\mathbf{0}$, 从而有 
$\overline{\mathbf{Q}}^{\mathrm{T}} \mathbf{F} \overline{\mathbf{Q}}=\left[\begin{array}{cc}\lambda \mathbf{I}_{3} & \mathbf{0} \\ \mathbf{0} & \mathbf{0}\end{array}\right] \in \mathbb{R}^{6 \times 6}$,

$\overline{\mathbf{Q}}^{\mathrm{T}} \mathbf{F} \mathbf{Q}=\left[\begin{array}{c}\mathbf{H}^{\mathrm{T}} \\ \mathbf{0}\end{array}\right] \in \mathbb{R}^{6 \times(3 n-6)}$,

其中, $\lambda(\mathbf{p}): \mathbb{R}^{3 n} \rightarrow \mathbb{R}, \mathbf{H}(\mathbf{p}): \mathbb{R}^{3 n} \rightarrow \mathbb{R}^{3 n \times 3}$ 定义为

$\lambda=\frac{1}{\left\|\mathbf{q}^{*}\right\|^{2}} \mathbf{q}^{* \mathrm{~T}} \mathbf{F} \mathbf{q}^{*}=\frac{1}{\left\|\mathbf{q}^{*}\right\|^{2}} \overline{\mathbf{q}}^{* \mathrm{~T}} \mathbf{F} \overline{\mathbf{q}}^{*}=\frac{1}{\left\|\overline{\mathbf{q}}^{*}\right\|^{2}} \overline{\overline{\mathbf{q}}}^{* \mathrm{~T}} \mathbf{F} \overline{\mathbf{q}}^{*}$,

$\mathbf{H}=\mathbf{Q}^{\mathrm{T}} \mathbf{F} \overline{\mathbf{Q}}_{1}$

又令 $\mathbf{p}_{1}=\left[\mathbf{p}_{11}{ }^{\mathrm{T}}, \mathbf{p}_{12}{ }^{\mathrm{T}}\right]^{\mathrm{T}}, \mathbf{p}_{11}, \mathbf{p}_{12} \in \mathbb{R}^{3}$. 将式(32)代入式 (31)中可得

$\left[\begin{array}{l}\ddot{\mathbf{p}}_{11} \\ \ddot{\mathbf{p}}_{12}\end{array}\right]=\varepsilon\left[\begin{array}{cc}\lambda \mathbf{I}_{3} & \mathbf{0} \\ \mathbf{0} & \mathbf{0}\end{array}\right]\left[\begin{array}{l}\mathbf{p}_{11} \\ \mathbf{p}_{12}\end{array}\right]+\varepsilon\left[\begin{array}{c}\mathbf{H}^{\mathrm{T}} \\ \mathbf{0}\end{array}\right] \mathbf{p}_{2}-k_{1}\left[\begin{array}{l}\dot{\mathbf{p}}_{11} \\ \dot{\mathbf{p}}_{12}\end{array}\right]$,

$\ddot{\mathbf{p}}_{2}=\varepsilon\left[\begin{array}{ll}\mathbf{H} & \mathbf{0}\end{array}\right]\left[\begin{array}{l}\mathbf{p}_{11} \\ \mathbf{p}_{12}\end{array}\right]+\mathbf{G p}_{2}-k_{1} \dot{\mathbf{p}}_{2}$

其中, $\mathbf{G}$ 定义为

$$
\mathbf{G}=k_{0} \mathbf{Q}^{\mathrm{T}} \mathbf{A} \mathbf{Q}+\varepsilon \mathbf{Q}^{\mathrm{T}} \mathbf{F} \mathbf{Q} \text {. }
$$

由于 $\mathbf{Q}^{\mathrm{T}} \mathbf{A Q}$ 为负定, $\|\mathbf{F}\|$ 有界, 对于足够小的 $\varepsilon, \mathbf{G}$ 仍 然为负定矩阵. 由式(35) 可得 $\ddot{\mathbf{p}}_{12}=-k_{1} \dot{\mathbf{p}}_{12}, \dot{\mathbf{p}}_{12} \rightarrow \mathbf{0}$, $\mathbf{p}_{12} \rightarrow \mathbf{p}_{12}{ }^{*}, \mathbf{p}_{12}{ }^{*}$ 为常数. 从而可得剩余的动力学方程:

$\ddot{\mathbf{p}}_{11}=\varepsilon \lambda \mathbf{p}_{11}+\varepsilon \mathbf{H}^{\mathrm{T}} \mathbf{p}_{2}-k_{1} \dot{\mathbf{p}}_{11}$,

$\ddot{\mathbf{p}}_{2}=\varepsilon \mathbf{H} \mathbf{p}_{11}+\mathbf{G} \mathbf{p}_{2}-k_{1} \dot{\mathbf{p}}_{2}$.

令 $\tau=\varepsilon t$, 则有 $\frac{\mathrm{d}}{\mathrm{d} t}=\varepsilon \frac{\mathrm{d}}{\mathrm{d} \tau}, \frac{\mathrm{d}^{2}}{\mathrm{~d} t^{2}}=\varepsilon^{2} \frac{\mathrm{d}^{2}}{\mathrm{~d} \tau^{2}}$, 则式(37)可以 表示成

$\ddot{\mathbf{p}}_{2}=\frac{1}{\varepsilon} \mathbf{H p}_{11}+\frac{1}{\varepsilon^{2}} \mathbf{G p}_{2}-\frac{k_{1}}{\varepsilon} \dot{\mathbf{p}}_{2}$,

$\varepsilon \ddot{\mathbf{p}}_{11}=\lambda \mathbf{p}_{11}+\mathbf{H}^{\mathrm{T}} \mathbf{p}_{2}-k_{1} \dot{\mathbf{p}}_{11}$,

其中, $\ddot{\mathbf{p}}_{11}, \ddot{\mathbf{p}}_{2}$ 表示对时间 $\tau$ 的微分.

$$
\begin{gathered}
\text { 令 } \mathbf{x}=\left[\begin{array}{l}
\mathbf{p}_{2} \\
\dot{\mathbf{p}}_{2}
\end{array}\right], \mathbf{z}=\left[\begin{array}{l}
\mathbf{p}_{11} \\
\dot{\mathbf{p}}_{11}
\end{array}\right], \text { 则式(38)可以表示成 } \\
\dot{\mathbf{x}}=\left[\begin{array}{cc}
\mathbf{0} & \mathbf{I}_{3 n-6} \\
\frac{\mathbf{G}}{\varepsilon^{2}} & -\frac{k_{1}}{\varepsilon} \mathbf{I}_{3 n-6}
\end{array}\right] \mathbf{x}+\left[\begin{array}{cc}
\mathbf{0} & \mathbf{0} \\
\frac{\mathbf{H}}{\varepsilon} & \mathbf{0}
\end{array}\right] \mathbf{z}, \\
\varepsilon \dot{\mathbf{z}}=\left[\begin{array}{cc}
\mathbf{0} & \mathbf{I}_{3} \\
\lambda \mathbf{I}_{3} & -k_{1} \mathbf{I}_{3}
\end{array}\right] \mathbf{z}+\left[\begin{array}{cc}
\mathbf{0} & \mathbf{0} \\
\mathbf{H}^{T} & \mathbf{0}
\end{array}\right] \mathbf{x} .
\end{gathered}
$$

可以看出系统式(38)为奇异摄动系统, 因此可以
使用引理4对其进行分析. 在使用引理4之前, 需做如 下说明. (1) 这里的分析是基于期望队形对应的位置 向量平衡点(包括平衡点绕 $z$ 轴的旋转、平移)以及智 能体最终的速度, 并不是引理 4 中的原点. 但这并不会 影响分析, 因为只需做一个简单的变换 $\mathbf{q}=\mathbf{q}-\mathbf{q}^{*}$, 便可 以把平衡点移到原点. (2) 原点 $\mathbf{q}=\mathbf{0}, \mathbf{v}=\mathbf{0}$ 为系统式(25) 的一个平衡点. 通过在这个平衡点附近线性化系统式 (25), 可以发现这个平衡点是不稳定的.

在期望的队形处 $\mathbf{q}=\mathbf{q}^{*}, \mathbf{v}=\mathbf{0}$, 由 $\mathbf{p}=\mathbf{U}^{\mathrm{T}} \mathbf{q}$ 可知, $\mathbf{p}_{11}=\mathbf{p}_{11}^{*}=\overline{\mathbf{Q}}_{1}^{\mathrm{T}} \mathbf{q}^{*}, \dot{\mathbf{p}}_{11}=\mathbf{0}, \mathbf{p}_{2}=\mathbf{Q}^{\mathrm{T}} \mathbf{q}^{*}=\mathbf{0}, \dot{\mathbf{p}}_{2}=\mathbf{0}$, 并且 由式(27)可知 $\mathbf{F}=\mathbf{0}$. 根据这些, 下面说明系统式(39)满足 引理4中的条件:

(1) 由于在期望的队形处 $\mathbf{F}=\mathbf{0}$, 由式(33)和(34)可知 $\lambda=0, \mathbf{H}=\mathbf{0} . \mathbf{x}=\left[\begin{array}{c}\mathbf{0} \\ \mathbf{0}\end{array}\right], \mathbf{z}=\left[\begin{array}{c}\mathbf{p}_{11}^{*} \\ \mathbf{0}\end{array}\right]$ 为系统式(39)的一个平衡点;

(2) 在式(39) 中令 $\varepsilon=0$, 得到似稳态方程 $\left[\begin{array}{cc}\mathbf{0} & \mathbf{I}_{3} \\ \lambda \mathbf{I}_{3} & -k_{1} \mathbf{I}_{3}\end{array}\right] \mathbf{z}+\left[\begin{array}{cc}\mathbf{0} & \mathbf{0} \\ \mathbf{H}^{T} & \mathbf{0}\end{array}\right] \mathbf{x}=\mathbf{0}$, 求解得到似稳态方程唯一 的孤立根: $\mathbf{z}=h(t, \mathbf{x})=\left[\begin{array}{cc}\mathbf{0} & \mathbf{0} \\ -\frac{\mathbf{H}^{\mathrm{T}}}{\lambda} & \mathbf{0}\end{array}\right] \mathbf{x}, \lambda \neq 0$, 且 $h(t, 0)=0$;

(3) 通过直接计算可以看出对于所有的 $\mathbf{x} \in \mathbb{R}^{6 n-12}$, $\mathbf{z} \in \mathbb{R}^{6}$, 式(39)等式右边的二阶导数均有界;

(4) 在式(39)中令 $\varepsilon=0, \mathbf{z}=\left[\begin{array}{cc}\mathbf{0} & \mathbf{0} \\ -\frac{\mathbf{H}^{\mathrm{T}}}{\lambda} & \mathbf{0}\end{array}\right] \mathbf{x}$, 得到 $\mathbf{G} \mathbf{p}_{2}=0$, 由于 $\mathbf{G}$ 为负定矩阵, 故 $\mathbf{p}_{2}=0, \dot{\mathbf{p}}_{2}=0$, 退化系 统 $\dot{\mathbf{x}}=f(t, \mathbf{x}, h(t, \mathbf{x}), 0)$ 在 $\mathbf{x}=0$ 处指数稳定;

(5) 在式(39)中令 $\varepsilon=0$, 得到边界层系统:

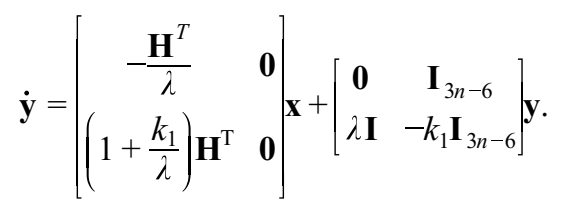

注意到 $\mathbf{x}=\left[\begin{array}{l}\mathbf{0} \\ \mathbf{0}\end{array}\right]$ 表明智能体达到期望的队形.

将 $\mathbf{x}=\left[\begin{array}{l}\mathbf{0} \\ \mathbf{0}\end{array}\right]$ 代入式(40)得到

$\dot{\mathbf{y}}=\left[\begin{array}{cc}\mathbf{0} & \mathbf{I}_{3 n-6} \\ \mathbf{0} & -k_{1} \mathbf{I}_{3 n-6}\end{array}\right] \mathbf{y}$. 
不难看出 $\mathbf{y}=\mathbf{0}$ 为边界层系统的一个全局指数稳定 平衡点.

又假设 $\mathbf{p}_{11}=\sigma \mathbf{p}_{11}{ }^{*}$, 其中 $\sigma>0$ 为队形的大小. 因而有

$$
\begin{cases}d_{i j}-d_{i j}^{*}<0, & \text { if } \sigma<1, \\ d_{i j}-d_{i j}^{*}=0, & \text { if } \sigma=1, \\ d_{i j}-d_{i j}^{*}>0, & \text { if } \sigma>1 .\end{cases}
$$

从 $\mathbf{F}$ 和 $\lambda$ 的定义及 $\sigma=\frac{\left\|\mathbf{p}_{11}\right\|}{\left\|\mathbf{p}_{11}^{*}\right\|}$ 可知

$\begin{cases}\lambda>0, & \text { if }\left\|\mathbf{p}_{11}\right\|<\left\|\mathbf{p}_{11}^{*}\right\|, \\ \lambda=0, & \text { if }\left\|\mathbf{p}_{11}\right\|=\left\|\mathbf{p}_{11}^{*}\right\|, \\ \lambda<0, & \text { if }\left\|\mathbf{p}_{11}\right\|>\left\|\mathbf{p}_{11}^{*}\right\| .\end{cases}$

式(43)表明预期的队形 $\mathbf{p}_{11}=\mathbf{p}_{11}{ }^{*}$ 为稳定的平衡点, 此时 $\lambda=0, \mathbf{H}^{\mathrm{T}}=\mathbf{0}$. 通过直接计算, 可知 $\lambda$ 在 $\sigma=1$ 处的斜率 为负数, 如图 3 所示, 因此 $\mathbf{p}_{11}=\mathbf{p}_{11}{ }^{*}$ 几乎全局指数稳定.

因此, 由引理 4 可知, 对于足够小的 $\varepsilon$, 期望的队形 是系统式(25)的一个指数稳定的平衡点. 又因为系统 式(25)在原点处存在一个不稳定的平衡点, 故这种指 数稳定性几乎是全局的.

注3 实际上只要取 $\varepsilon<\left|\frac{\lambda_{\text {max }}}{\mu_{\text {max }}}\right|$ 即可, 其中 $\mu_{\text {max }}=2(n$ -1) $f_{\max }, f_{\max }$ 表示函数 $f$ 的一个上界, $\lambda_{\max }$ 表示矩阵 $\mathbf{A}$ 的最 大非零特征值.

\section{5 领航者-跟随者网络下的二阶集群系统立 体编队控制}

领航者-跟随者网络下的集群系统编队中，领航者 指的是多智能体在编队航行过程中控制整个编队行进 路线的那部分智能体，领航者的运动轨迹或运动方程 一般可以通过航迹规划预先给定，跟随者通过控制其 与领航者之间的角度和距离, 保持期望的编队队形.

\section{1 控制律设计}

在本节中，考虑从具有无向感知拓扑的二阶集群 系统中选取 $n \geq 2$ 个智能体作为领航者，领航者静止在 目标位置, 剩余 $n_{f}$ 个智能体作为跟随者, 跟随者跟随领 航者形成特定的立体队形.

在所设计的二阶集群系统编队保持控制律式(24)

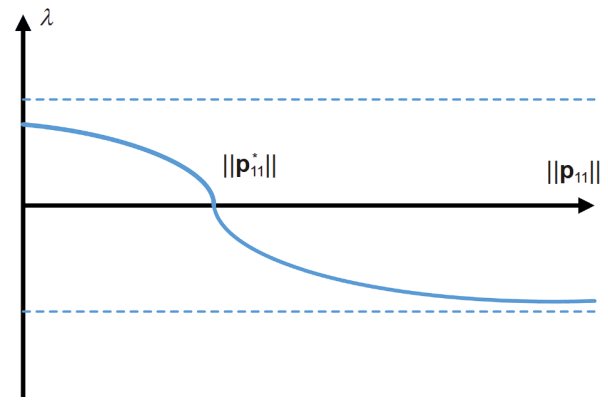

图 3 (网络版彩图) $\lambda$ 数值与 $\left\|\mathbf{p}_{11}\right\|$ 的关系曲线

Figure 3 (Color online) Relationship curve between the value $\lambda$ with $\left\|\mathbf{p}_{11}\right\|$.

的基础上, 考虑将智能体 $i$ 的控制律设计为

$\mathbf{v}_{i}=0, \quad i=1,2 \ldots, n_{\ell}$,

$\mathbf{u}_{i}=\sum_{j \in \mathcal{N}_{i}} \mathbf{L}_{i j}\left(\mathbf{q}_{j}-\mathbf{q}_{i}\right)+\varepsilon * f\left(d_{i j}-d_{i j}{ }^{*}\right)\left(\mathbf{q}_{j}-\mathbf{q}_{i}\right)-\gamma \mathbf{v}_{i}$,

$i=n-n_{f}+1, \ldots, n$,

其中, $n_{\ell}+n_{f}=n, \varepsilon$ 和 $\gamma$ 为增益.

系统的闭环动力学方程可以表示成

$\left[\begin{array}{c}\dot{\mathbf{q}} \\ \dot{\mathbf{v}}\end{array}\right]=\left[\begin{array}{cc}0 & \mathbf{I}_{3 n} \\ \mathbf{L}+\varepsilon \mathbf{F} & -\gamma \mathbf{I}_{3 n}\end{array}\right]\left[\begin{array}{l}\mathbf{q} \\ \mathbf{v}\end{array}\right]$,

其中,

$\mathbf{L}=\left[\begin{array}{cc}\mathbf{0}_{3 n_{\ell} \times 3 n_{\ell}} & \mathbf{0}_{3 n_{\ell} \times 3 n_{f}} \\ \mathbf{L}_{f l} & \mathbf{L}_{f f}\end{array}\right] \in \mathbb{R}^{3 n \times 3 n}$.

而 $\mathbf{L}$ 和3.1节中的增益矩阵 $\mathbf{A}$ 定义相同, $\mathbf{F}$ 和4.2节中 的 $\mathbf{F}$ 定义相同, 均具有块拉普拉斯结构.

$\mathbf{F}=\left[\begin{array}{cc}\mathbf{0}_{3 n_{\ell} \times 3 n_{\ell}} & \mathbf{0}_{3 n_{\ell} \times 3 n_{f}} \\ \mathbf{F}_{f l} & \mathbf{F}_{f f}\end{array}\right] \in \mathbb{R}^{3 n \times 3 n}$.

\section{2 稳定性证明}

定理5 考虑具有动力学式(3)和控制律式(44)的二 阶集群系统, 如果设计的增益矩阵 $\mathbf{L}$ 满足定理 1 中的条 件(1), 并且 $\mathbf{L}_{f f}$ 为对称负定矩阵, 则存在 $\varepsilon^{*}>0, \gamma^{*}>0$ 使得 对于所有的 $0<\varepsilon<\varepsilon^{*}, \gamma>\gamma^{*}$, 期望的编队队形是一个几乎 全局指数稳定的平衡点, 即跟随者能够跟随领航者达 到期望的队形.

证明 令 $\widetilde{\mathbf{q}}=\mathbf{q}^{-} \mathbf{q}^{*}, \widetilde{\mathbf{v}}=\mathbf{v}-\mathbf{v}^{*}$, 分别表示编队过程 中的状态误差向量, 其中 $\mathbf{q}^{*}, \mathbf{v}^{*}$ 分别表示智能体的期望 
位置向量与速度向量, $\mathbf{v}^{*}=\mathbf{0}$. 从而有

$\dot{\tilde{\mathbf{q}}}=\widetilde{\mathbf{v}}$

$\dot{\tilde{\mathbf{v}}}=(\mathbf{L}+\varepsilon \mathbf{F})\left(\widetilde{\mathbf{q}}+\mathbf{q}^{*}\right)-\gamma \widetilde{\mathbf{v}}$.

易知 $\widetilde{\mathbf{q}}$ 和 $\widetilde{\mathbf{v}}$ 的前 $3 n_{\ell}$ 个元素均为 0 , 令 $\widetilde{\mathbf{q}}_{\mathbf{F}}$ 和 $\widetilde{\mathbf{v}}_{\mathbf{F}}$ 分别表 示向量 $\mathbf{q}$ 和 $\widetilde{\mathbf{v}}$ 的后 $3 n_{f}$ 个元素, $\mathbf{q}_{\mathbf{L}}^{*}$ 和 $\mathbf{q}_{\mathbf{F}}^{*}$ 分别表示 $\mathbf{q}^{*}$ 的前 $3 n_{\ell}$ 个元素和后 $3 n_{f}$ 个元素, 则式(48)可以写成

$\dot{\widetilde{\mathbf{q}}}_{\mathbf{F}}=\widetilde{\mathbf{V}}_{\mathbf{F}}$,

$\dot{\widetilde{\mathbf{v}}}_{\mathbf{F}}=\left(\mathbf{L}_{f f}+\varepsilon \mathbf{F}_{f f}\right) \widetilde{\mathbf{q}}_{\mathbf{F}}+\varepsilon\left(\mathbf{F}_{f f} \mathbf{q}_{\mathbf{L}}^{*}+\mathbf{F}_{f f} \mathbf{q}_{\mathbf{F}}^{*}\right)-\gamma \widetilde{\mathbf{v}}_{\mathbf{F}}$.

注意到 $\mathbf{L}_{f f}$ 为负定矩阵, $\| \mathbf{F}_{f f} \mid$ 有界, 则存在足够小的 $\varepsilon^{*}$, 当 $0<\varepsilon<\varepsilon^{*}$ 时, $\mathbf{L}_{f f}+\varepsilon \mathbf{F}_{f f}$ 仍然为负定矩阵.

定义一个备选李雅普诺夫函数:

$V_{2}=-\frac{1}{2} \widetilde{\mathbf{q}}_{\mathbf{F}}^{\mathrm{T}}\left(\mathbf{L}_{f f}+\varepsilon \mathbf{F}_{f f}\right) \widetilde{\mathbf{q}}_{\mathbf{F}}+\frac{1}{2} \widetilde{\mathbf{v}}_{\mathbf{F}}^{\mathrm{T}} \widetilde{\mathbf{v}}_{\mathbf{F}} \geq 0$.

对 $V_{2}$ 求导可得

$$
\begin{aligned}
\dot{V}_{2}= & -\widetilde{\mathbf{q}}_{\mathbf{F}}^{\mathrm{T}}\left(\mathbf{L}_{f f}+\varepsilon \mathbf{F}_{f f}\right) \widetilde{\mathbf{v}}_{\mathbf{F}}+\widetilde{\mathbf{v}}_{\mathbf{F}}^{\mathrm{T}}\left[\left(\mathbf{L}_{f f}+\varepsilon \mathbf{F}_{f f}\right) \widetilde{\mathbf{q}}_{\mathbf{F}}\right. \\
& \left.+\varepsilon\left(\mathbf{F}_{f t} \mathbf{q}_{\mathbf{L}}^{*}+\mathbf{F}_{f f} \mathbf{q}_{\mathbf{F}}^{*}\right)-\gamma \widetilde{\mathbf{v}}_{\mathbf{F}}\right] .
\end{aligned}
$$

而 $\mathbf{L}_{f f}, \mathbf{F}_{f f}$ 为对称矩阵, 故:

$$
\begin{aligned}
\dot{V}_{2} & =\widetilde{\mathbf{v}}_{\mathbf{F}}^{\mathrm{T}}\left[\varepsilon\left(\mathbf{F}_{f f} \mathbf{q}_{\mathbf{L}}^{*}+\mathbf{F}_{f f} \mathbf{q}_{\mathbf{F}}^{*}\right)-\gamma \widetilde{\mathbf{v}}_{\mathbf{F}}\right] \\
& =\varepsilon \widetilde{\mathbf{v}}_{\mathbf{F}}^{\mathrm{T}}\left(\mathbf{F}_{f t} \mathbf{q}_{\mathbf{L}}^{*}+\mathbf{F}_{f f} \mathbf{q}_{\mathbf{F}}^{*}\right)-\gamma \widetilde{\mathbf{v}}_{\mathbf{F}}^{\mathrm{T}} \widetilde{\mathbf{v}}_{\mathbf{F}} \\
& \leq \varepsilon\left\|\widetilde{\mathbf{v}}_{\mathbf{F}}\right\|\left(\left\|\mathbf{F}_{f t}\right\| \mathbf{q}_{\mathbf{L}}^{*}\|+\| \mathbf{F}_{f f}\left\|\mathbf{q}_{\mathbf{F}}^{*}\right\|\right)-\gamma\left\|\widetilde{\mathbf{v}}_{\mathbf{F}}\right\|^{2} \\
& =\left\|\widetilde{\mathbf{v}}_{\mathbf{F}}\right\|_{2}\left[\varepsilon\left(\left\|\mathbf{F}_{f f}\right\|\left\|\mathbf{q}_{\mathrm{L}}^{*}\right\|+\left\|\mathbf{F}_{f f}\right\|\left\|\mathbf{q}_{\mathbf{F}}^{*}\right\|\right)-\gamma\left\|\widetilde{\mathbf{v}}_{\mathbf{F}}\right\|\right] .
\end{aligned}
$$

由于 $\| \mathbf{F}_{f f l} \mid$ 和 $\| \mathbf{F}_{f f} \mid$ 有界, $\left\|\mathbf{q}_{\mathbf{L}}{ }^{*}\right\|$ 和 $\left\|\mathbf{q}_{\mathbf{F}}{ }^{*}\right\|$ 为已知, 且当达 到期望队形时 $\left\|\mathbf{F}_{f l}\right\|=0,\left\|\mathbf{F}_{f f}\right\|=0$, 结合 4.2 节中对编队保持 控制策略的分析可知, 存在足够小的 ${ }^{*}$ 及足够大的 $\gamma^{*}$, 使得当 $0<\varepsilon<\varepsilon_{\varepsilon}^{*}, \gamma>\gamma^{*}$ 时, $\dot{V}_{2} \leq 0$ 恒成立. 再由拉萨尔不变 性定理可知, $\mathbf{q}_{\mathbf{F}} \rightarrow \mathbf{0}, \mathbf{v}_{\mathbf{F}} \rightarrow \mathbf{0}$, 因而 $\mathbf{q}_{\mathbf{F}} \rightarrow \mathbf{q}_{\mathbf{F}}{ }^{*}, \mathbf{v}_{\mathbf{F}} \rightarrow \mathbf{0}$, 即 $\mathbf{q} \rightarrow \mathbf{q}^{*}, \mathbf{v} \rightarrow \mathbf{0}$, 即跟随者跟随领航者达到期望的立体 队形.

\section{3 增益矩阵 $\mathrm{L}$ 的优化求解}

结合定理 5 中对系统的稳定性分析, 进一步设计了 领航者-跟随者网络下增益矩阵 $\mathbf{L}$ 的优化求解算法, 如 表3所示.
表 3 领航者-跟随者网络下增益矩阵 $\mathbf{L}$ 的优化求解算法

Table 3 Algorithm for optimal calculation of gain matrix Lunder leader-follower networks

算法3: 领航者-跟随者网络下增益矩阵 $\mathbf{L}$ 的优化求解

输入: 期望队形坐标 $\mathbf{q}^{*}$.

输出: 增益矩阵 $\mathbf{L} \in \mathbb{R}^{3 n \times 3 n}$.

步骤1: 令 $\mathbf{L}=\left[\begin{array}{cc}\mathbf{0}_{3 n \ell^{\times 3 n_{\ell}}} & \mathbf{0}_{3 n_{\ell} \times 3 n_{f}} \\ \mathbf{L}_{f l} & \mathbf{L}_{f f}\end{array}\right] \in \mathbb{R}^{3 n \times 3 n}$, 其中 $\mathbf{L}_{f f}$ 为对称负定矩阵.

步骤2: 令 $\mathbf{N}=\left[\mathbf{q}^{*}, \overline{\mathbf{q}}^{*}, \overline{\overline{\mathbf{q}}}^{*}, 1_{x}, 1_{y}, 1_{z}\right] \in \mathbb{R}^{3 n \times 6}$.

步骤3: 使用SDP求解器求解式 $\begin{gathered}\mathbf{L}=\underset{a_{i j},{ }_{i j}, c_{i j}}{\operatorname{argmax}} \\ \text { subject to }\end{gathered} \quad \lambda_{\min }\left(-\mathbf{L}_{f f}\right)$
$\mathbf{L N}=0$ 所述的半

正定规划问题.

\section{6 仿真实验及分析}

\section{1 二阶集群系统立体编队控制仿真}

本节将对于二阶集群系统立体编队控制进行仿真 验证, 选取 5 个二阶积分器模型的智能体, 将其分别编 号为 $1,2, \ldots, 5$, 并且假定 5 个智能体的期望队形为金字 塔队形. 实验采用所设计的控制律式(24), 将智能体的 队形大小固定并且加入避碰算法 2 , 给定激活阈值 $d=5$, 安全半径 $r=2$, 控制增益 $k_{0}=k_{1}=\varepsilon=1$, 所有智能体的初始 速度为 0 , 随机设置 5 个智能体的全局初始位置为

$\mathbf{q}_{0}=\left[\begin{array}{ccccc}12.9329 & 8.2202 & 10.2059 & 1.1734 & 0.7176 \\ 6.6439 & 8.5029 & 5.5707 & 6.8453 & 11.0739 \\ 3.0000 & 4.0000 & 5.0000 & 6.0000 & 8.0000\end{array}\right]$.

给定 5 个智能体的预期金字塔队形为

$\mathbf{q}_{s}=\left[\begin{array}{llllc}0 & 2 & 2 & 0 & 1 \\ 0 & 0 & 2 & 2 & 1 \\ 0 & 0 & 0 & 0 & \sqrt{2}\end{array}\right] \times 7.5$.

给定 5 个智能体的拓扑结构:

$\mathbf{M}=\left[\begin{array}{lllll}0 & 1 & 0 & 1 & 1 \\ 1 & 0 & 1 & 0 & 1 \\ 0 & 1 & 0 & 1 & 1 \\ 1 & 0 & 1 & 0 & 1 \\ 1 & 1 & 1 & 1 & 0\end{array}\right]$.

根据给定的拓扑结构, 首先可以使用MATLAB CVX凸优化工具箱利用算法1求解得出增益矩阵 $\mathbf{A}$. 图4 给出了 5 个智能体分别在 $t=0,3,6 \mathrm{~s}$ 以及 $15 \mathrm{~s}$ 时刻达到 期望队形并保持的仿真过程, 图5(a)给出了仿真过程 中智能体间的距离变化曲线, 图中平行于 $x$ 轴的线表示 

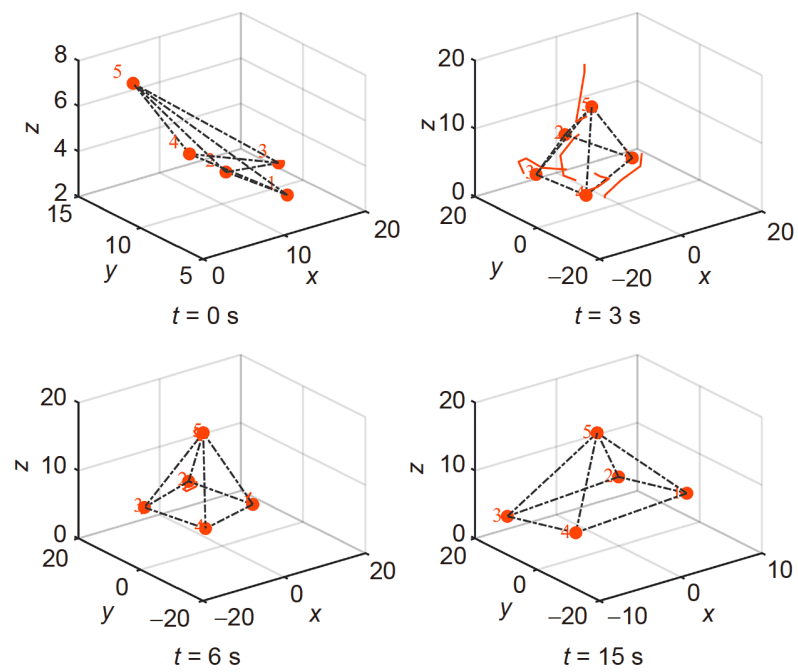

图 4 (网络版彩图)5个智能体形成金字塔队形仿真过程

Figure 4 (Color online) Evolutionary trajectories of 5 agents to form a pyramid shape.
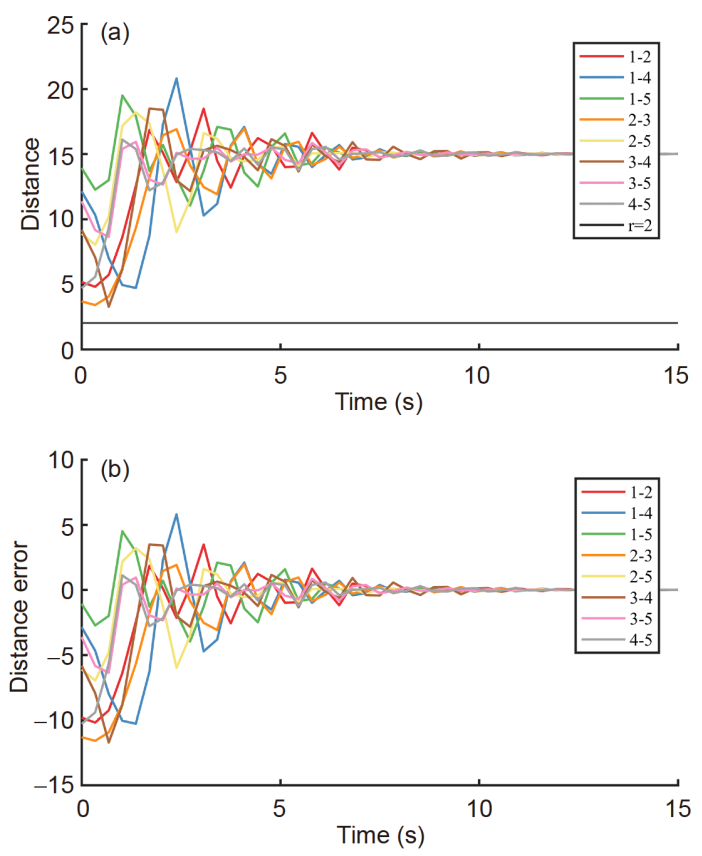

图 5 (网络版彩图)智能体间的距离(a)和误差(b)变化曲线

Figure 5 (Color online) Distances between neighboring agents. (a) Distance curves; (b) distance error curves.

安全半径 $r=2$, 图5(b)显示了智能体间的距离与期望距 离之间的误差变化曲线. 结合图4和 5 可以看出，初始 时刻 $t=0 \mathrm{~s}$ 智能体位于随机位置, 此后直至约 $t=5 \mathrm{~s}$ 时间 段金字塔队形初步形成， $t=5 \sim 10 \mathrm{~s}$ 时间段智能体在小 范围进行移动, 而 $t=10 \mathrm{~s}$ 之后金字塔队形能够稳定保
持, 且智能体间距离误差保持为 0 . 通过观察仿真结果, 发现智能体编队最终收玫到了期望的金字塔队形，队 形的大小满足了期望的固定值, 并且在编队过程中智 能体间没有发生碰撞, 整个仿真实验验证了所设计的 编队控制律式(24)和避碰算法2的正确性和有效性.

\section{2 领航者-跟随者网络下的二阶集群系统立体编 队控制仿真}

本节将对于领航者-跟随者网络下的二阶集群系 统立体编队控制进行仿真验证，假设领航者和跟随者 均为二阶积分器模型, 其中 $n=8$ 个领航者, 整个仿真过 程中分别静止在立方体的 8 个顶点上, 另外 $n_{f}=5$ 个跟随 者初始速度均被设置为 0 , 最终期望跟随者编队形成一 个包含在领航者立方体队形内的金字塔队形. 仿真采 用跟随者的编队控制律式(44), 设定的控制增益为 $\varepsilon=1, \gamma=1$.

假设 8 个领航者一直分别保持静止在相应的全局 位置:

$\mathbf{q}_{\ell 0}=\left[\begin{array}{cccccccc}4 & 4 & 12 & 12 & 4 & 4 & 12 & 12 \\ 12 & 4 & 4 & 12 & 12 & 4 & 4 & 12 \\ 4 & 4 & 4 & 4 & 12 & 12 & 12 & 12\end{array}\right]$.

给定 5 个跟随者的全局初始位置为

$\mathbf{q}_{f 0}=\left[\begin{array}{ccccc}0.0000 & 2.6734 & 8.7934 & 13.2534 & 4.5600 \\ 2.3000 & 15.2500 & 0.2879 & 2.7665 & 6.7820 \\ 10.2675 & 12.2665 & 2.7500 & 13.2500 & 14.2476\end{array}\right]$.

设定领航者与跟随者间的拓扑结构为完全图，而 跟随者间的拓扑结构如仿真图6中所示, 利用所设计 的算法3, 使用MATLAB CVX凸优化工具箱可以求解 得到增益矩阵 $\mathbf{L}$. 图6给出了在给定 8 个领航者的立方 体编队之后, 5 个跟随者分别在 $t=0,5,10$ 以及 $15 \mathrm{~s}$ 时刻 收敛到立方体内部形成期望的金字塔队形并保持的仿 真过程. 而图7中显示了智能体间的距离与期望距离之 间的误差变化曲线, 距离误差在 $t=10 \mathrm{~s}$ 左右收敛并保持 为零, 曲线共有 48 条, 故在此忽略标注. 结合图6和7可 以看出, 初始时刻 $t=0 \mathrm{~s}$ 跟随者位于随机位置, 此后直至 约 $t=5 \mathrm{~s}$ 时间段跟随者编队在立方体内部的金字塔队形 初步形成, $t=5 \sim 10 \mathrm{~s}$ 时间段智能体在小范围进行移动, 而 $t=10 \mathrm{~s}$ 之后金字塔队形能够稳定保持. 可以观察得 出跟随者编队最终收敛并保持到了期望的包含在领航 者立方体内部的金字塔队形，所设计的编队控制律式 

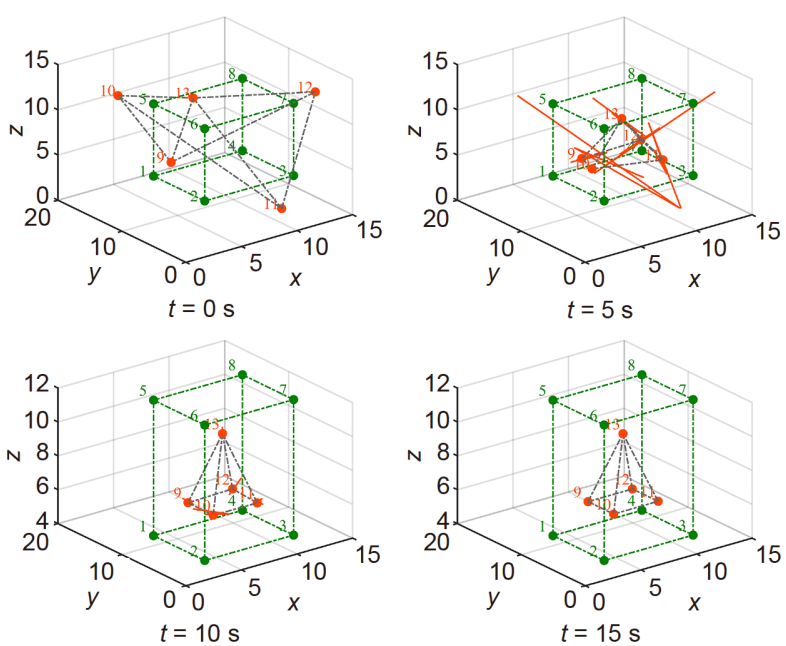

图 6 (网络版彩图) 5 个跟随者跟踪 8 个领航者形成金字塔队 形仿真过程

Figure 6 (Color online) Evolutionary trajectories of 5 followers tracking 8 leaders to form a pyramid shape.

(44)的正确性和有效性得到了证实.

\section{7 总结}

假设集群系统中相对位置信息可知，且内部无相 互通信，本文提出了一种基于增益矩阵的分布式编队 控制策略，使得二阶动力学系统编队在立体空间内，

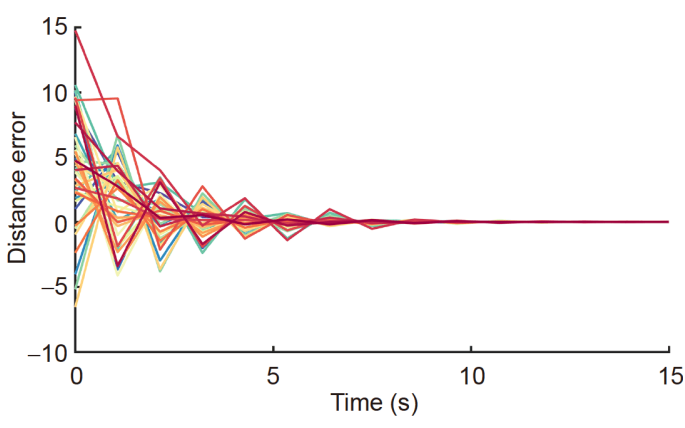

图 7 (网络版彩图)智能体间的距离误差变化曲线

Figure 7 (Color online) Curves of distance errors between neighboring agents.

能够生成与保持. 首先，设计的半正定规划算法能够 对增益矩阵进行优化求解; 提出的编队控制律对扰 动、饱和输入等具有较好的鲁棒性. 其次, 采用启发 式的避碰算法，使得避免智能体在收敛到期望队形的 过程中相互之间的碰撞; 利用带有距离约束的编队保 持控制策略，使得整个编队能够保持固定大小的队形. 最后，将控制律扩展至领航者-跟随者网络，使得跟随 者能够跟随领航者达到预期的编队队形. 结合文献 [30]中的改进梯度控制律，本文所提出的编队控制策 略能够进一步应用于独轮车(unicycle)、前轮驱动车 (front-wheel drive car)、后轮驱动车(rear-wheel drive car)等非完整约束动力学模型.

致谢 感谢Aerospace Controls Laboratory at the Massachusetts Institute of Technology的Dr. Kaveh Fathian对于本文研究 方法给予的探讨和支持.

\section{参考文献}

1 Keller J, Thakur D, Likhachev M, et al. Coordinated path planning for fixed-wing UAS conducting persistent surveillance missions. IEEE Trans Automat Sci Eng, 2017, 14: 17-24

2 Thomas J, Welde J, Loianno G, et al. Autonomous flight for detection, localization, and tracking of moving targets with a small quadrotor. IEEE Robot Autom Lett, 2017, 2: 1762-1769

3 Dorling K, Heinrichs J, Messier G G, et al. Vehicle routing problems for drone delivery. IEEE Trans Syst Man Cybern Syst, 2017, 47: 70-85

4 Wang Z, Schwager M. Kinematic multi-robot manipulation with no communication using force feedback. In: 2016 IEEE International Conference on Robotics and Automation (ICRA). Stockholm, 2016. 427-432

5 Saldana D, Gabrich B, Whitzer M, et al. A decentralized algorithm for assembling structures with modular robots. In: 2017 IEEE International Conference on Intelligent Robots and Systems (IROS). Vancouver, 2017. 2736-2743

6 Bähnemann R, Schindler D, Kamel M, et al. A decentralized multi-agent unmanned aerial system to search, pick up, and relocate objects. In: 2017 IEEE International Symposium on Safety, Security and Rescue Robotics (SSRR). Shanghai, 2017. 123-128

7 Oh K K, Park M C, Ahn H S. A survey of multi-agent formation control. Automatica, 2017, 53: 424-440 
8 Shen D, Wei R X, Ru C J. Digital-pheromone-based control method for UAV swarm search (in Chinese). J Syst Eng Electron, 2013, 35: 591-596 [沈东, 魏瑞轩, 茹常剑. 基于数字信息素的无人机集群搜索操控方法.系统工程与电子技术. 2013, 35: 591-596]

9 Wang X K, Li X, Zheng Z Q. Survey of developments on multi-agent formation control related problems (in Chinese). Control Decision, 2013, 28: 1601-1613 [王祥科, 李迅, 郑志强. 多智能体系统编队控制相关问题研究综述. 控制与决策, 2013, 28: 1601-1613]

10 Hyun N P, Vela P A, Verriest E I. Collision free and permutation invariant formation control using the root locus principle. In: IEEE 2016 American Control Conference (ACC). Boston, 2016. 2572-2577

11 Motoyama T, Cai K. Top-down synthesis of multi-agent formation control: An eigenstructure assignment based approach. In: IEEE 2017 American Control Conference (ACC). Seattle, 2017. 259-264

12 Montijano E, Zhou D, Schwager M, et al. Distributed formation control without a global reference frame. In: 2014 IEEE American Control Conference (ACC). Portland, 2014. 3862-3867

13 Zhao S Y, Zelazo D. Bearing rigidity and almost global bearing-only formation stabilization. IEEE Trans Automat Contr, 2015, 61: 1255-1268

14 Park M C, Sun Z Y, Anderson B D O, et al. Distance-based control of $K_{n}$ formations in general space with almost global convergence. IEEE Trans Automat Contr, 2018, 63: 2678-2685

15 Weinstein A, Cho A, Loianno G, et al. Visual inertial odometry swarm: An autonomous swarm of vision-based quadrotors. IEEE Robot Autom Lett, 2018, 3: 1801-1807

16 Aranda M, López-Nicolás G, Sagüés C, et al. Coordinate-free formation stabilization based on relative position measurements. Automatica, 2015, 57: $11-20$

17 Tian Y P, Wang Q. Global stabilization of rigid formations in the plane. Automatica, 2013, 49: 1436-1441

18 Deghat M, Bishop A N. Distributed shape control and collision avoidance for multi-agent systems with bearing-only constraints. In: 2015 IEEE European Control Conference (ECC). Linz, 2015. 2342-2347

19 Trinh M H, Zhao S Y, Sun Z Y, et al. Bearing-based formation control of a group of agents with leader-first follower structure. IEEE Trans Automat Contr, 2018, 64: 598-613

20 Lin Z Y, Wang L L, Chen Z Y, et al. Necessary and sufficient graphical conditions for affine formation control. IEEE Trans Automat Contr, 2016, 61: $2877-2891$

21 Peinado H G D M, Jayawardhana B, Cao M. Distributed algorithm for controlling scale-free polygonal formations. In: 2017 World Congress of the International Federation of Automatic Control (IFAC). Toulouse, 2017. 1760-1765

22 Han T R, Lin Z Y, Zheng R H, et al. A Barycentric coordinate-based approach to formation control under directed and switching sensing graphs. IEEE Trans Cybern, 2018, 48: 1202-1215

23 Zhang M M, Wei C. Multi-UAV formation control method based on edge Laplacian consensus (in Chinese). Sci Sin Tech, 2017, 47: 259-265 [张 苗苗, 魏晨. 基于边Laplacian一致性的多无人机编队控制方法. 中国科学: 技术科学, 2017, 47: 259-265]

$24 \mathrm{Xu} \mathrm{Y,} \mathrm{Li} \mathrm{D} \mathrm{Y,} \mathrm{Luo} \mathrm{D} \mathrm{L,} \mathrm{et} \mathrm{al.} \mathrm{Affine} \mathrm{formation} \mathrm{maneuver} \mathrm{tracking} \mathrm{control} \mathrm{of} \mathrm{multiple} \mathrm{second-order} \mathrm{agents} \mathrm{with} \mathrm{time-varying} \mathrm{delays.} \mathrm{Sci} \mathrm{China}$ Tech Sci, 2019, 62: 665-676

$25 \mathrm{Xu} \mathrm{Y,} \mathrm{Luo} \mathrm{D} \mathrm{L,} \mathrm{You} \mathrm{Y} \mathrm{C,} \mathrm{et} \mathrm{al.} \mathrm{Affine} \mathrm{transformation} \mathrm{based} \mathrm{formation} \mathrm{maneuvering} \mathrm{for} \mathrm{discrete-time} \mathrm{directed} \mathrm{networked} \mathrm{systems.} \mathrm{Sci} \mathrm{China}$ Tech Sci, 2020, 63: 73-85

26 Fathian K, Safaoui S, Summers T H, et al. Robust distributed planar formation control for higher-order holonomic and nonholonomic agents. ArXiv: 1807.11058, 2018

27 Boyd S, Vandenberghe L. Convex Optimization-Boyd and Vandenberghe. Cambridge: Cambridge University Press, 2004. 127-136

28 Liberzon D. Switching in Systems and Control. Boston: Springer Science \& Business Media, 2012. 17-20

29 Fathian K, Rachinskii D I, Spong M W, et al. Distributed formation control via mixed Barycentric coordinate and distance-based approach. In: 2019 American Control Conference (ACC). Philadelphia, 2019. 51-58

30 Zhao S Y, Dimarogonas D V, Sun Z Y, et al. A general approach to coordination control of mobile agents with motion constraints. IEEE Trans Automat Contr, 2018, 63: 1509-1516 


\title{
A gain matrix approach for robust distributed 3D formation control with second order swarm systems
}

\author{
XU Yang, LUO DeLin, ZHOU LuPeng, SHAO Jiang \& YOU YanCheng \\ School of Aerospace Engineering, Xiamen University, Xiamen 361102, China
}

This article addresses the problem of shape formation and control in robust distributed second order swarm systems in 3D space. The control strategy described is based on a gain matrix approach and can be executed using only relative position measurements in each agent's local coordinate frame, which does not rely on inter-agent communication. First, a 3D formation shaping control method for second order swarm systems is proposed, and global stability is proved by constructing the candidate Lyapunov function. The gain matrix can be calculated by convex optimization, and the disturbance and input saturation is eliminated by the robustness of the proposed control law. Additionally, a heuristic algorithm for collision avoidance is introduced during the formation shaping process. After shaping the desired formation, the formation control law with required distances is proposed for maintaining a fixed formation shape in swarm systems. The control laws are then extended to 3D second order systems with leader-follower networks. Finally, the correctness of theoretical results is verified using simulations.

distributed formation control, second-order swarm systems, robustness, collision avoidance, leader-follower network

doi: 10.1360/SST-2019-0258 